\title{
Analysis of the Registration Information on Interventions of Acupuncture and Moxibustion Trials in the International Clinical Trials Registry Platform
}

\author{
Yali Liu $\left(\mathbb{D},{ }^{1,2,3}\right.$ Wenjie Chen, ${ }^{4}$ Yingxin Tan, ${ }^{4}$ Xingyue Yang $\left(\mathbb{D},{ }^{5}\right.$ Jia Liu $\left(\mathbb{D},{ }^{1}\right.$ Tingting Lu, ${ }^{3}$ \\ Shiyan Yan, ${ }^{1}$ Liyun He, ${ }^{1}$ and Baoyan Liu ${ }^{6}{ }^{6}$ \\ ${ }^{1}$ Institute of Basic Research in Clinical Medicine, China Academy of Chinese Medical Sciences, Beijing 100700, China \\ ${ }^{2}$ Postdoctoral Research Station, China Academy of Chinese Medical Sciences, Beijing 100700, China \\ ${ }^{3}$ Evidence Based Medicine Center, School of Basic Medical Sciences, Lanzhou University, Lanzhou, Gansu 730000, China \\ ${ }^{4}$ The First Clinical Medical College of Lanzhou University, Lanzhou, Gansu 730000, China \\ ${ }^{5}$ Beijing University of Chinese Medicine, Beijing 100700, China \\ ${ }^{6}$ China Academy of Chinese Medical Sciences, Beijing 100700, China
}

Correspondence should be addressed to Baoyan Liu; baoyanjournal@163.com

Received 3 August 2017; Accepted 15 November 2017; Published 15 January 2018

Academic Editor: Caigan Du

Copyright (C) 2018 Yali Liu et al. This is an open access article distributed under the Creative Commons Attribution License, which permits unrestricted use, distribution, and reproduction in any medium, provided the original work is properly cited.

\begin{abstract}
Purpose. To analyze and compare the clinical registration information about acupuncture and moxibustion for intervention characteristics. Methods. Clinical trials from the International Clinical Trials Registry Platform of the World Health Organization in acupuncture and moxibustion were comprehensively collected from 2013 to 2015; data were independently screened and extracted by two retrievers, and relevant data involving either basic descriptions or intervention characteristics were analyzed. Results. 425 acupuncture and moxibustion registered clinical trials were included; 88.00\% (374/425) were designed as controlled studies, among which $38.59 \%$ (164/425) had sham acupuncture as the control group. The most common diseases were pain-related at approximately $19.29 \%(82 / 425)$ of trials. Reports on the intervention information in these acupuncture and moxibustion clinical studies were not sufficiently presented; these reports included the reporting of names of points (39.8\%), the method of needle stimulation (32.5\%), needle type (29.6\%), needle retention time (34.1\%), the number of treatment sessions $(22.4 \%)$, and the frequency and duration of treatment sessions (38.1\%). Conclusion. The registration information for the clinical trials of acupuncture and moxibustion was quite low according to this investigational study. Steps should be taken to improve the quality of acupuncture and moxibustion registration information.
\end{abstract}

\section{Introduction}

Acupuncture and moxibustion have been practiced in China for more than 3000 years and are considered to be complementary and alternative therapy [1]. Benefits from these therapies include relative safety, effectiveness, feasibility, and economic wellbeing. Since 1979, the World Health Organization (WHO) has promulgated 43 diseases with indications applicable to acupuncture and moxibustion [2]. Therefore, these traditional Chinese therapies have become publicly acceptable in clinical practice in the West [3].
Since the 20th century, acupuncture and moxibustion clinical trials have progressed into a bustling and booming era not only in the quantity of studies but also in quality; in addition, the diseases included in clinical trials have expanded over these decades [4,5]. Many problems and concerns manifested during this time period; for example, there was a shortage of well-designed and strictly implemented clinical studies [6]. Evidence from these clinical studies could not be cited and generalized to improve acupuncture and moxibustion practice. The barrier has been diminishing with solid evidence. 
In November 2004 in Mexico City, the WHO was regarded as the lead organization in establishing the International Clinical Trials Registry Platform (ICTRP) [7]. This milestone event was a consensus decision during the Ministerial Summit on Health Research [8]. It was in May 2007 when the ICTRP officially broke ground [9], and the importance of trial registration has been gradually accepted [10-12]. To date, 16 data providers, including the United States and China, have been authorized as the first registration institutions of the WHO ICTRP [13]. In May 2007, the WHO Trial Registration Data Set (TRDS) was announced, setting a minimum of 20 items for trial registration [14]. International clinical trial registration has been implemented to a large extent to improve the quality of clinical trials, as well as form a transparent presentation of trial progress and execution [15].

This study aims to collect and analyze the 20 items of the WHO TRDS and other registry information from the 16 clinical trial register centers of the WHO ICTRP; provide an overview of the registered acupuncture-moxibustion clinical trials for manipulation specifications and reporting performance; and propose the necessity to establish the Acupuncture-Moxibustion Clinical Trial Registry (AMCTR) center.

\section{Materials and Methods}

2.1. Inclusion/Exclusion Criteria. All acupuncture and moxibustion clinical trials registered in the WHO ICTRP were eligible for inclusion. No criteria for disease were applied, nor were criteria applied for participant age, sex, and ethnicity; the studied interventions were acupuncture and moxibustion, including Filiform needle, percussopunctator, Auricular Acupuncture, or transcutaneous electrical acupoint stimulation, indicated as the major medical treatment. The control group for any comparison was placebo, no intervention, or another intervention. There was no definition for endpoint outcomes. Exclusion criteria included incomplete registry information, no information indicating that acupuncture or moxibustion had been used to intervene in the disease studied or that acupuncture or moxibustion were only used as a secondary concomitant therapy. Acupuncture, needling, acupressure, moxibustion, auriculotherapy, and acupoint were used as keyword search terms, regardless of the initial registry language.

\subsection{Database Sources. Data from the WHO ICTRP (http://} apps.who.int/trialsearch/Default.aspx) registry records between 2013 and 2015 were used. This registry platform included Australia, New Zealand, China, Korea, United States, India, Cuba, Germany, Iran, United Kingdom (UK), Japan, Pan African Region, Sri Lanka, Netherlands, Belgium, and the European Union (EU) Region. The specific names of the registries are as follows: Australian New Zealand Clinical Trials Registry (ANZCTR, Australia, and New Zealand), Chinese Clinical Trial Register (ChiCTR, China), Clinical Research Information Service (CRIS, Republic of Korea), Clinical Trials.gov (United States), Clinical Trials Registry, India (CTRI,
India), Cuban Public Registry of Clinical Trials (RPCEC, Cuba), German Clinical Trials Register (DRKS, German), Iranian Registry of Clinical Trials (IRCT, Iran), ISRCTN.org (British), Japan Primary Registries Network (JPRN, Japan), Pan African Clinical Trial Registry (PAC-TR, Africa), Sri Lanka Clinical Trials Registry (SLCTR, Sri Lanka), the Netherlands National Trial Register (NTR, Netherlands), Brazilian Clinical Trials Registry (ReBec, Brazil), EU Clinical Trials Register (EU-CTR, EU), and Thai Clinical Trials Registry (TCTR, Thailand).

2.3. Data Extraction. Using a predefined data extraction form that collected information for this study, two evaluators independently extracted data (Wenjie Chen and Yingxin Tan); disagreements in the data extraction were resolved by another evaluator (Yali Liu) after further consultation. The content of the data extraction forms was categorized into the following sections.

2.3.1. Basic Registry Information on the 16 Clinical Trial Registry Centers. This information included the name of the registry platform, nationality/region, website, language, the date in which the platform was established, the setup and disposition of the 16 first-rate study centers worldwide, and the 20 items in the World Health Organization (WHO) Trial Registration Data Set (TRDS).

The categories for the registry information were formulated by the 16 ICTRP clinical study centers; these included the registered title for the platform, update time, registry time, status of the registry number, information on the applicant, approval-related report from the institutional ethics board, information on the study-center that conducted the designated trials, funding and financial resources, study design (disease, study category/type, purpose, design, methodology, inclusion and exclusion criteria, interventions, outcomes, and endpoints), study time (initiation, duration, and close-out), designated study sites, human histology samples collected, subject recruitment and enrollment, study data management institution, and any commissioned/contracted data analysis companies/agents.

2.3.2. Basic Information Registered Clinical Trials in the Acupuncture and Moxibustion Fields. These fields involved registry time, the name of the clinical trials registry platform, study type, sample size, the object disease/symptoms, intervention(s), and endpoint outcomes.

2.3.3. Specific Information on Interventions in Registered Clinical Trials in the Acupuncture and Moxibustion Fields. Based on the Standards for Reporting Interventions in Clinical Trials of Acupuncture (STRICTA) [16], the data extraction form was used to investigate the manipulation procedures. The information collected included the number of needle insertions per subject per session, names (or location if no standard name) of points used, depth of insertion, response sought, needle stimulation, needle retention time, needle type, and the registry information on sham acupuncture. 
TABLE 1: The characteristic information of platforms in ICTRP.

\begin{tabular}{lccc}
\hline Platform & Countries/District & Internet site & $\begin{array}{c}\text { Language } \\
\text { The time of } \\
\text { establishment }\end{array}$ \\
\hline ANZCTR & Australia/New Zealand & http://www.anzctr.org.au/ & English \\
ChiCTR & China & http://www.chictr.org.cn/ & English, Chinese \\
CRIS & Kore & http://cris.nih.go.kr/ & English, Korean \\
Clinical Trials.gov & United States & http://www.clinicaltrials.gov/ & English \\
CTRI & India & http://www.ctri.nic.in/ & English \\
RPCEC & Cuba & http://registroclinico.sld.cu/ & English, Spanish \\
DRKS & Germany & http://www.germanctr.de/ & English, German \\
IRCT & Iran & http://www.irct.ir/ & English, Arabic \\
ISRCTN.org & United Kingdom & http://www.isrctn.org/ & English \\
JPRN & Japan & http://rctportal.niph.go.jp/ & English, Japanese \\
PAC-TR & Africa & http://www.pactr.org/ & English \\
SLCTR & Sri Lanka & http://www.slctr.lk/ & 2005 \\
NTR & Netherlands & http://www.trialregister.nl/ & English \\
ReBec & Brazil & http://www.ensaiosclinicos.gov.br/ & English, Portuguese \\
EU-CTR & Europe & https://www.clinicaltrialsregister.eu/ & English \\
TCTR & Thailand & http://www.clinicaltrials.in.th/ & English, Thai
\end{tabular}

2.4. Data Analysis. Microsoft Excel (Version 2007) was used to analyze the coded data items for any statistically significant differences. Categorical data is presented as number $(n)$ and percent (\%).

\section{Results}

3.1. Basic Information of the Registered Clinical Trial Centers of ICTRP. To date, 16 registries have been designated by the WHO ICTRP as first-rate clinical trial registry centers which cover all six continents. These are summarized in Table 1.

3.2. Categorization of the Registry Information Designated by the ICTRP Clinical Trial Registry Centers. There are 20 items in the TRDS stipulated by the WHO ICTRP that are reported by the first sixteen registry platforms, as shown in Table 2. Moreover, specific registry information from these centers supplements the fundamental TRDS. In Table 3, some supplemental data are included, except for the 20 items in the WHO TRDS; in this study, a total of 45 registry items are included. It is notable to mention that the ANZCTR had defined more than 30 items for its registry information; other centers have sets of items that vary from 15 to 25 .

3.3. Basic Registry Information for Acupuncture and Moxibustion Clinical Trials. We reviewed 425 acupuncture and moxibustion clinical trials that had completed registration between 2013 and 2015. Among them, 124 (29.18\%) were registered in 2013, 123 (28.94\%) were registered in 2014, and $178(41.89 \%)$ were registered in 2015 . The studies that were registered in the WHO ICTRP were mainly interventional studies $(89.18 \%, 379 / 425)$; only $3.53 \%$ (14/425) were observational studies; $88.00 \%(374 / 425)$ were controlled studies and 164 (38.59\%) were compared with sham acupuncture; 187 and 50 studies recruited participants from China (including Hong Kong and Taiwan) and the United States, respectively. The largest sample size was 1000 subjects (Table 4). Clinical Trials.gov, ChiCTR, and CRIS had the most clinical studies (Table 5). Pain symptoms were the main indication for treatment with acupuncture or moxibustion, comprising 82 (19.29\%) of the studies; the other diseases and symptoms were neoplasm $(7.29 \%, 31 / 425)$, stroke $(4.00 \%, 17 / 425)$, arthritis $(3.53 \%, 15 / 425)$, depression $(3.06 \%, 13 / 425)$, and insomnia $(2.59 \%, 11 / 428)$ (Table 6).

3.4. Characteristics of the Acupuncture and Moxibustion Trials in the WHO ICTRP. As summarized in Table 7, there were no sufficiently specific descriptions of acupuncture and moxibustion manipulation or the procedures in these reports of the clinical trials registered between 2013 and 2015. These trials mainly covered Acu-point [39.8\% (169/425)], the method for acupuncture stimulation [32.4\% (138/425)], the instrumental needle type [29.6\% (126/425)], needling keep-in time [34.1\% (145/425)], unit number for acupuncture therapy $[22.4 \%(95 / 425)]$, therapeutic frequency, and the timing of each acupuncture scheme/regimen $[38.1 \%$ $(162 / 425)]$. Among the countries of recruitment in these platforms, China represented 1.6\% (3/187), 34.8\% (65/187), $10.7 \%$ (20/187), 8.0\% (15/187), 39.6\% (74/187), 29.4\% (55/187), $17.1 \%(32 / 187), 16.0 \%$ (30/187), and $32.1 \%$ (60/187), respectively. Fewer trials reported other details of the acupuncture intervention. For example, only $11.8 \%$ (50/425) of the trials reported anchoring depth, 8.9\% (38/425) reported if a somatic response was triggered (such as specific self-sensual response from a receiver), and $4.9 \%(21 / 425)$ reported the number of needles. The manipulation in these registered 


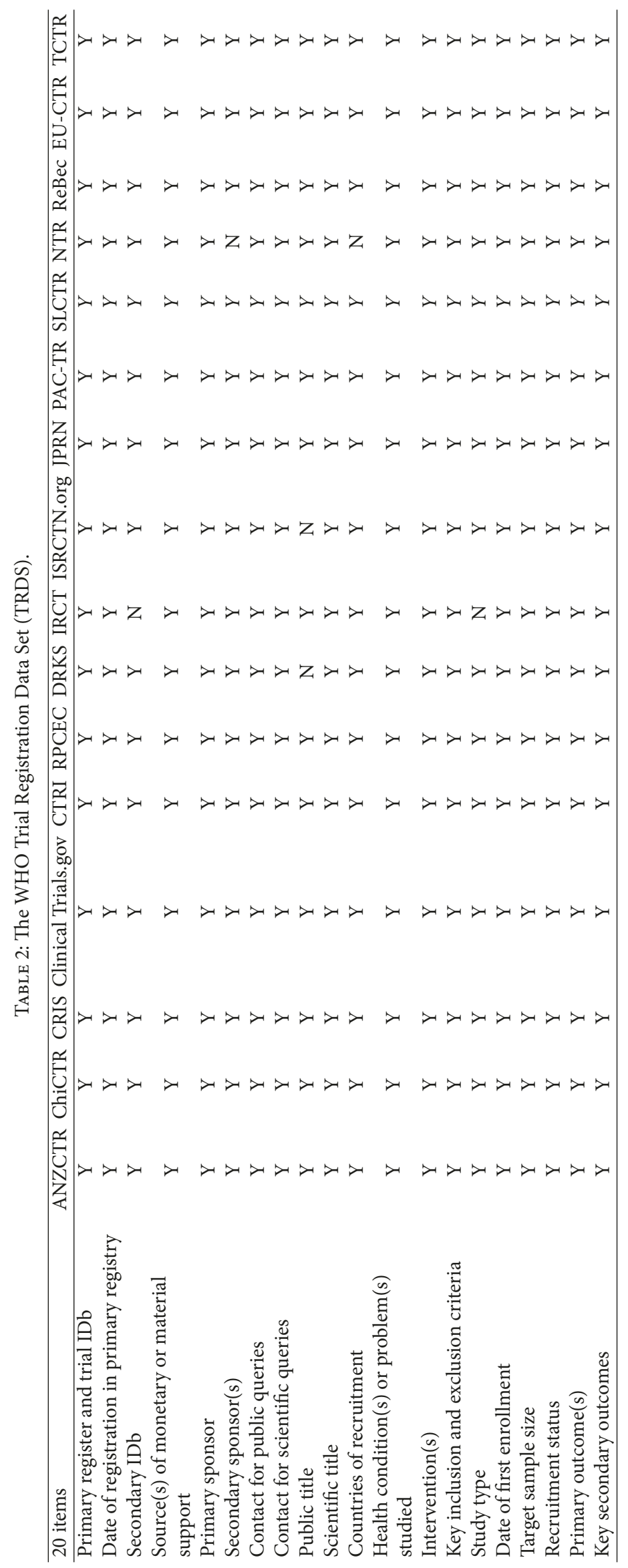




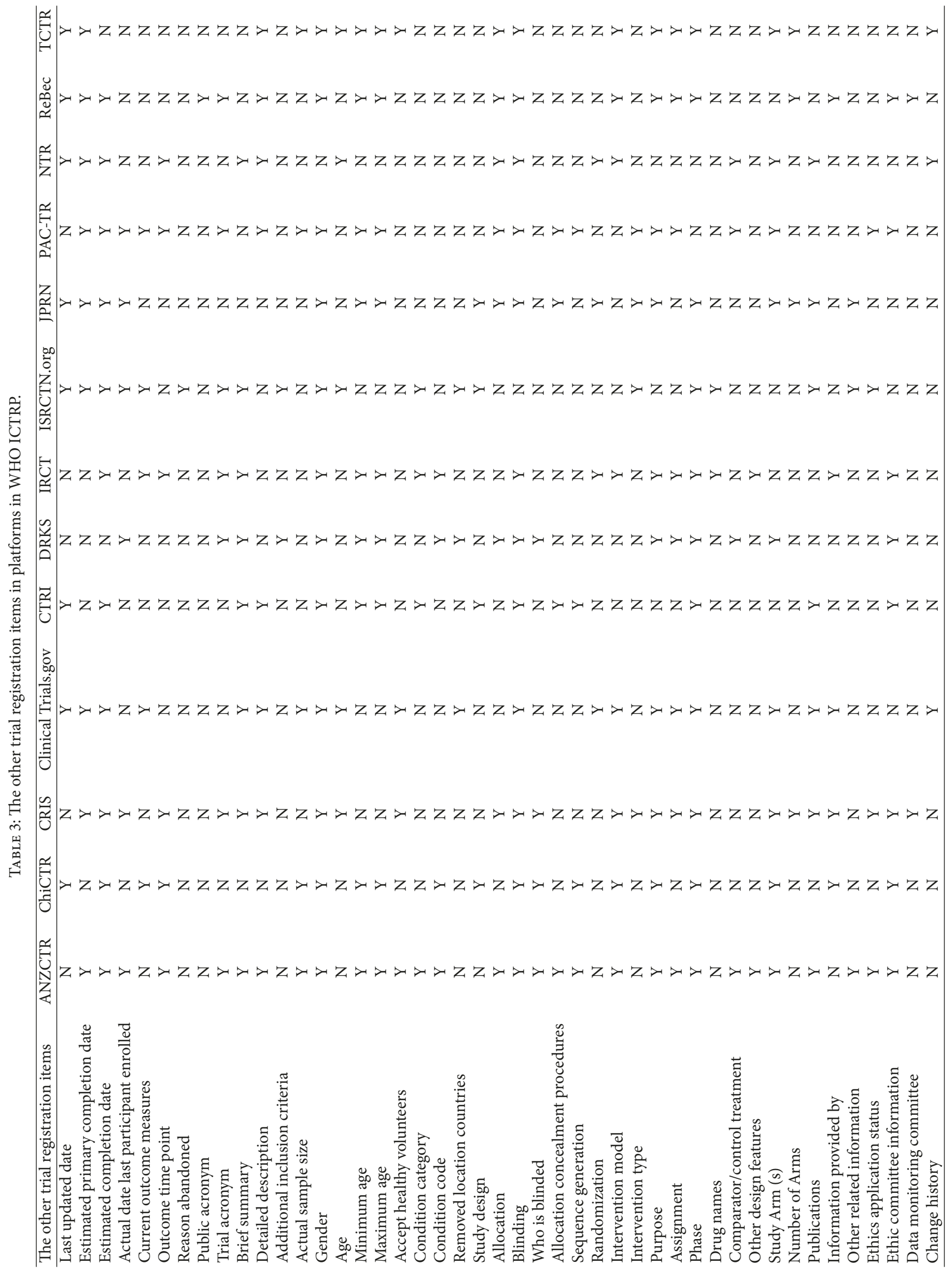


TABLE 4: The number of clinical trials of acupuncture-moxibustion in WHO ICTRP.

\begin{tabular}{|c|c|c|}
\hline Category & Characteristic & $\begin{array}{l}\text { Number of } \\
n=425(\%)\end{array}$ \\
\hline \multirow{3}{*}{$\begin{array}{l}\text { Register time } \\
\text { (year) }\end{array}$} & 2013 & 124 \\
\hline & 2014 & 123 \\
\hline & 2015 & 178 \\
\hline \multirow{3}{*}{ Study type } & Intervention study & 379 \\
\hline & Observation study & 14 \\
\hline & Others & 32 \\
\hline \multirow{4}{*}{ Study design } & With control group & 374 \\
\hline & Sham acupuncture in control group & 164 \\
\hline & $\begin{array}{l}\text { Acupuncture versus nonacupuncture } \\
\text { (e.g., pharmacologic treatment, physiotherapy) }\end{array}$ & 163 \\
\hline & The other treatment program & 97 \\
\hline \multirow{5}{*}{ Countries of recruitment } & China & $187^{*}$ \\
\hline & United States & 50 \\
\hline & Korea & 47 \\
\hline & Other Countries & 120 \\
\hline & Unclear & 21 \\
\hline Sample size & Maximum/minimum & $1000 / 4$ \\
\hline
\end{tabular}

${ }^{*}$ Including Hongkong and Taiwan.

TABLE 5: The number of clinical trials of acupuncture-moxibustion in 16 countries in WHO ICTRP.

\begin{tabular}{|c|c|c|c|c|c|c|}
\hline & Countries/district & Internet site & $\begin{array}{l}\text { The number of } \\
\text { clinical trials in } \\
\text { acupuncture }\end{array}$ & $\begin{array}{l}\text { The number of } \\
\text { clinical trials In } \\
\text { moxibustion }\end{array}$ & $\begin{array}{l}\text { The } \\
\text { others }\end{array}$ & $\begin{array}{c}\text { Total } \\
\text { number }\end{array}$ \\
\hline \multicolumn{7}{|l|}{ Platform } \\
\hline Clinical Trials.gov & United States & http://www.clinicaltrials.gov/ & $178\left(176+2^{*}\right)$ & $3\left(1+2^{*}\right)$ & 22 & 201 \\
\hline ChiCTR & China & http://www.chictr.org.cn/ & 99 & 3 & 4 & 106 \\
\hline CRIS & Kore & http://cris.nih.go.kr/ & $28\left(27+1^{*}\right)$ & $2\left(1+1^{*}\right)$ & 1 & 30 \\
\hline IRCT & Iran & http://www.irct.ir/ & 13 & 0 & 8 & 21 \\
\hline ANZCTR & $\begin{array}{c}\text { Australia/New } \\
\text { Zealand }\end{array}$ & http://www.anzctr.org.au/ & 8 & 0 & 4 & 12 \\
\hline ISRCTN.org & United Kingdom & http://www.isrctn.org/ & 16 & 0 & 1 & 17 \\
\hline JPRN & Japan & http://rctportal.niph.go.jp/ & $16\left(14+2^{*}\right)$ & $4\left(1+2^{*}\right)$ & 0 & 18 \\
\hline DRKS & Germany & http://www.germanctr.de/ & 4 & 0 & 1 & 5 \\
\hline ReBec & Brazil & http://www.ensaiosclinicos.gov.br/ & 5 & 0 & 1 & 6 \\
\hline TCTR & Thailand & http://www.clinicaltrials.in.th/ & 6 & 0 & 0 & 6 \\
\hline NTR & Netherlands & http://www.trialregister.nl/ & 1 & 0 & 0 & 1 \\
\hline PAC-TR & Africa & http://www.pactr.org/ & 1 & 0 & 0 & 1 \\
\hline CTRI & India & http://www.ctri.nic.in/ & 1 & 0 & 0 & 1 \\
\hline Total & & & & & & 425 \\
\hline
\end{tabular}

${ }^{*}$ Report both acupuncture and moxibustion in titles.

studies was mainly manual acupuncture $(55.06 \%, 234 / 425)$; in the others it was electrical stimulation $(22.59 \%, 96 / 425)$, acupressure $(4.23 \%, 18 / 425)$, auricular acupuncture $(3.53 \%$, $15 / 425)$, dry needling $(4.70 \%, 20 / 425)$, laser acupuncture $(4.23 \%, 18 / 425)$, moxibustion $(2.35 \%, 10 / 425)$, auricular acupressure $(0.47 \%, 2 / 425)$, thermotherapy $(0.70 \%, 3 / 425)$, and pharmaco-acupuncture $(0.23 \%, 1 / 425)$ (Table 8$)$.
Among the 425 trials, 164 (38.59\%) trials used sham acupuncture as a control, but specific information, such as the application materials $(0.61 \%, 1 / 164)$, needle stimulation $(14.02 \%, 23 / 164)$, depth of insertion $(11.59 \%, 19 / 164)$, and needle retention time $(0.61 \%, 1 / 164)$, was reported at a low rate; $61(32.70 \%)$ trials did not report any details about the sham acupuncture (Table 9 ). 
TABLE 6: The condition focus on in the trails in ICTRP.

\begin{tabular}{|c|c|c|c|c|}
\hline Condition focused on in the studies & Acupuncture & Moxibustion & The others & Total number \\
\hline Pain & 75 & 1 & 6 & 82 \\
\hline Cancer & 28 & 0 & 3 & 31 \\
\hline Stroke & $17\left(16+1^{*}\right)$ & $1^{*}$ & 0 & 17 \\
\hline Arthritis & 11 & 0 & 4 & 15 \\
\hline Depression & 11 & 0 & 2 & 13 \\
\hline Insomnia & 11 & 0 & 0 & 11 \\
\hline Polycystic ovarian syndrome & 9 & 0 & 1 & 10 \\
\hline Obesity & 7 & 0 & 2 & 9 \\
\hline Hypertension & 6 & 0 & 1 & 7 \\
\hline The others & $208\left(202+6^{*}\right)$ & $11\left(5+6^{*}\right)$ & 24 & 237 \\
\hline Total & 376 & 6 & 43 & $425^{\#}$ \\
\hline
\end{tabular}

* Report both acupuncture and moxibustion in titles. ${ }^{\#}$ Seven trials with 2 diseases in titles (2 with pain + cancer, 2 with cancer + insomnia, 2 with stroke + depression, 2 with depression + insomnia).

\section{Discussion}

4.1. Current Status of the Registration Information in the WHO ICTRP. In this overview, we have compared the registry items of the 16 clinical centers that were designated according to the recommendations of the WHO ICTRT. Generally, the minimum 20 items in the WHO TRDS were followed; however, information outside of these 20 items varied widely between different countries. The main information reported was a brief summary, purpose, trial completion time (registry update date, time to trial termination), methodologic details (inclusion criteria, age of the subjects, study phase, and design including groups/distribution/blinding of subjects and blinding of groups/interventions/drug names), and other information (dropouts, ethical issues, data monitoring committee, and updates on important information).

4.2. Deficiencies in the Characteristics of Clinical Trials of Acupuncture and Moxibustion Registered in the ICTRP. Acupuncture and moxibustion are a core part of traditional Chinese medicine and are also very important in complementary and alternative medicine. Compared with surgery and other modern medical interventions, acupuncture and moxibustion are characterized by obvious differences. To standardize clinical study reporting on acupuncture and moxibustion, STRICTA and STRICTOM (Standards for Reporting Interventions in Clinical Trials of Moxibustion) have been issued as an extended section in the CONSORT statement [17-19].

Six criteria have been set as STRICTA items: acupuncture rationale, details of needling, treatment regimen, other components of treatment, practitioner background, and control or comparator interventions [17]. In this study, a data extraction form was designed based on the STRICTA to collect and analyze the registration information. As the results indicated, necessary information, such as instrumental needle type, name of points used, depth of insertion, method of needle stimulation, needle retention time, response sought, and treatment sessions, was incompletely reported (less than $40 \%$ according to our analysis). However, this information needs to be described in detail because it is an important component of integrative therapy, directly influencing the authenticity and reliability of studies.

Controlled studies are fundamental to assessing effects in clinical trials. The purpose of controlled studies is to minimize confounding factors and provide a reliable, cogent conclusion. Because of their unique manipulation process, acupuncture and moxibustion have encountered difficulties in developing and validating placebo needles as appropriate controls [20]. Researchers designate sham acupuncture as a control group, such as sham treatment with invasive skin or needling at inappropriate points or at nonacupuncture points, needling with blunt needles, sham laser, and sham electroacupuncture [16, 21-24]. However, the rationale for these simulation designs and their anticipated placebo-effect is closely related to conscientious pseudo-manipulation and, consequently, rigid implementation. Detailed reports are needed about sham acupuncture materials, sham-acupoint, or locations without standard names, manipulation methods, and procedures (stimulation methods, depth of insertion). Moreover, the rationale for sham-needling or its purported placebo-effect should also be explained; otherwise, the reader of the study cannot comprehend or be persuaded to trust the sham-needling maneuvers to enhance the accuracy of measuring intervention effectiveness. Among the 164 trials reporting the use of sham acupuncture, some necessary information such as materials, stimulation methods, depth of insertion, and duration are not available because of the low incidence of reporting.

The reporting rate of the characteristics of acupuncture and moxibustion in the WHO ICTRP for both Chinese and clinical trials and those from other countries was low. The reasons may be as follows: (1) all 16 clinical trials registry centers were founded based on the mode or convention of Westernmedicine-oriented registry format design, which may not have sufficiently considered the characteristics/specifications of acupuncture and moxibustion; (2) clinical trials reported and delineated acupuncture interventions in a meticulous manner, and completing registration information was done with little detail; (3) the inclusion and exclusion criteria for the clinical trials, such as the PICO, have not been included 


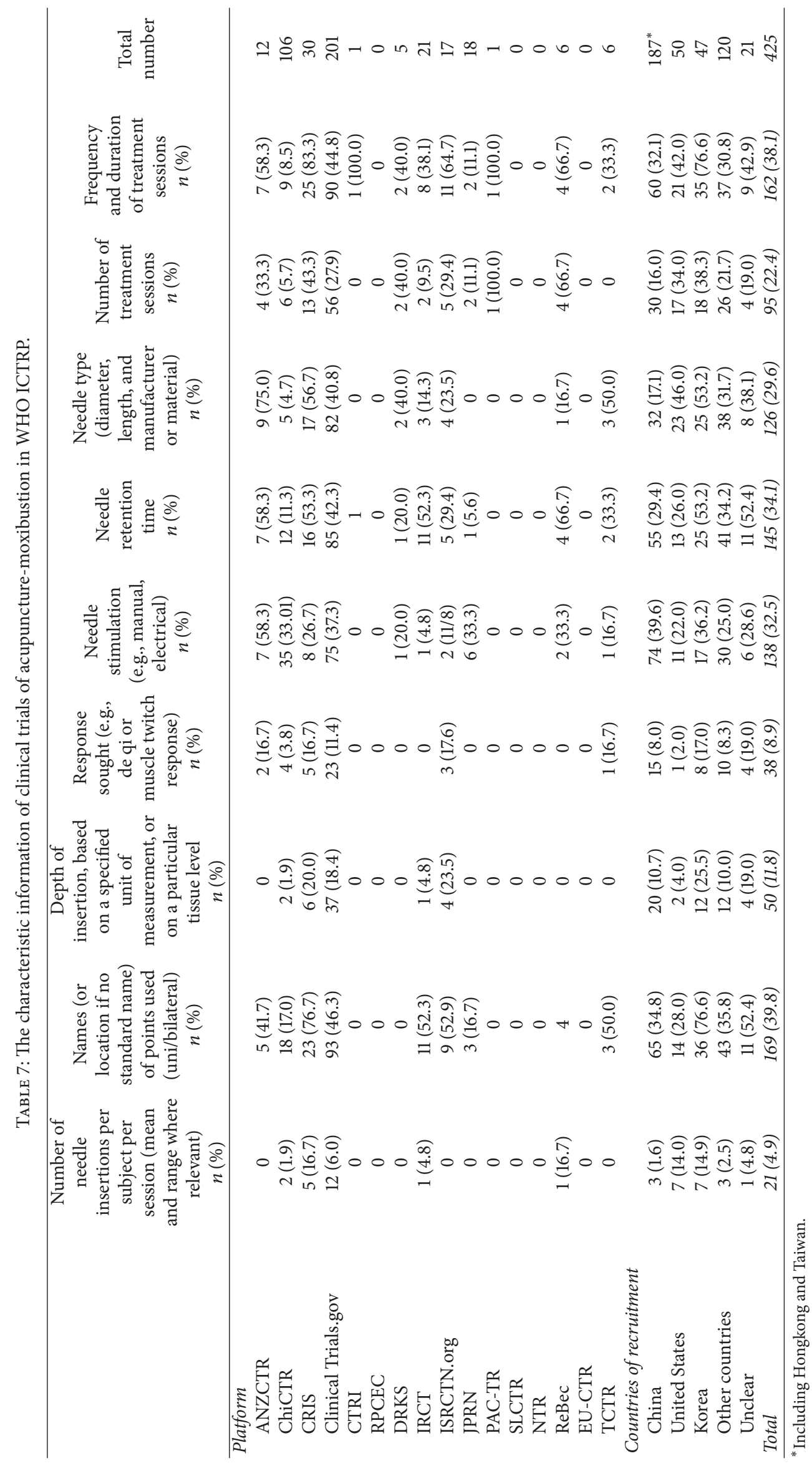




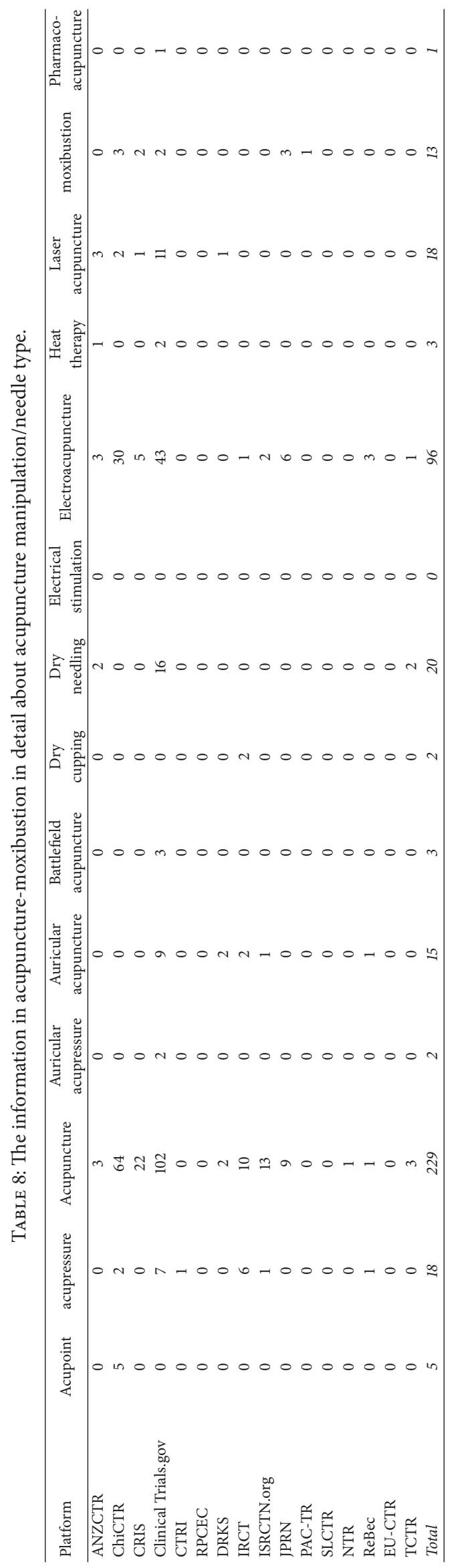




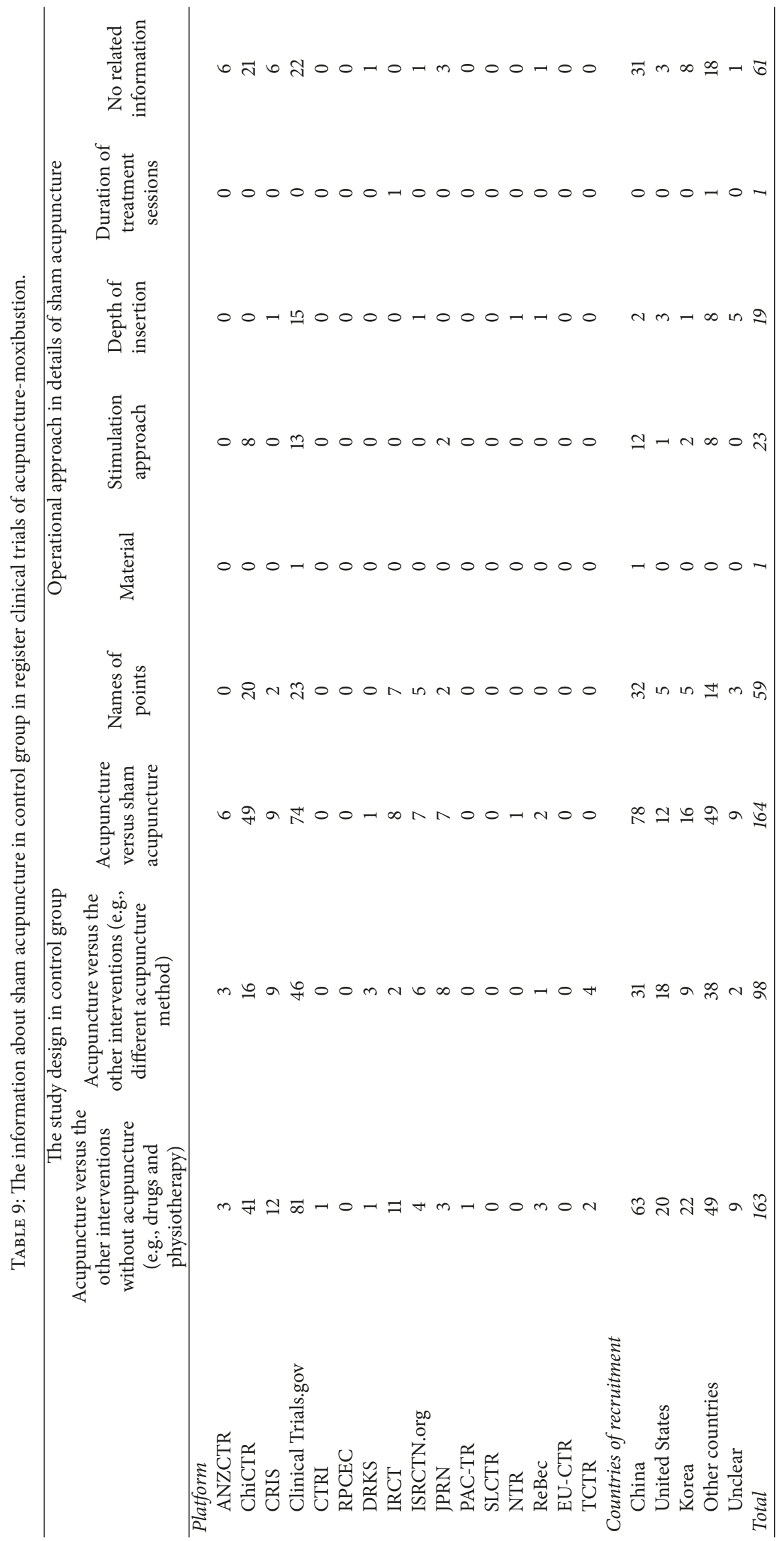


in the registration forms even though they might be well described in the trial protocols because of the limitation of the module in clinical register platform.

To summarize, some necessary information may have been missed in the registered clinical trials. This implies that steps of the registration process may not have been highly valued and, consequently, led to deficiencies in these reports.

\subsection{Considerable Work Needs to Be Done to Improve Method-} ological and Reporting Quality of Acupuncture and Moxibustion Clinical Trials. As developments in clinical epidemiology and evidence-based medicine continue, some methods of interventional investigation to guide clinical practice have been modified/updated into more incisive study designs. Clinical studies in acupuncture and moxibustion have been affected by the introduction of some clinical study methods, as demonstrated by the increased number of trials and the reporting quality; although there was steady progress, some remarkable redirected attention could be identified.

However, problems in practice and in studies inhibit substantial improvement and adaptation to modern medicine. Overall, rigidly controlled studies resulting in a highly credible publication comprised only a small number of the total [6]. Second, many trials have deficiencies and deviate from the clinical study methodology, even these that were labelled as systematic assessments and randomized controlled trials. Specific examples include lack of statistical calculation of sample size, incomplete analysis of baseline data, fragmented reporting on follow-up, or insufficiently scheduled visits, which can seriously undermine the reliability of clinical evidentiary studies [25]. Third, there are still large gaps between the clinical evidence and the clinical practice of acupuncture and moxibustion.

Therefore, a challenging and long-term goal to be undertaken is promoting the standardization of acupuncture and moxibustion clinical trial registries. The current principles and guidelines of institutions for clinical registration trials are skewed in favor of western medical drugs or surgical interventions, ignoring the special characteristics of acupuncture and moxibustion, which are founded on the MeridianCollateral Theory.

The Acupuncture-Moxibustion Clinical Trials Registry (AMCTR), making the second-tier of the WHO ICTRP, has been established to regulate clinical trials of acupuncture and moxibustion [26]. This registry center will be obliged to improve the global mechanism, promote data collection, facilitate data sharing, regulate specific trial studies, and assure publicized honesty and integrity of acupuncture and moxibustion studies. It will also provide a registry service in Chinese regions, as well as for worldwide interest.

4.4. Limitations. Limitations include the following: (1) the study was only based on data collection from 2013 to 2015 from the WHO ICTRP register trials in acupuncture and moxibustion; (2) the main focus is on registering information from designated sources instead of acquiring specific study protocols; and (3) incomplete information from these registries could influence the results.

\section{Conflicts of Interest}

The authors declare that there are no conflicts of interest regarding the publication of this article.

\section{Acknowledgments}

This study was supported and funded by the program of "the 12th Five-Year" National Science and Technology Pillar Program (2012BAI24B01) by the Ministry of Science and Technology of the People's Republic of China.

\section{References}

[1] Y. Zhang, J. J. Xing, J. Li, B. Y. Zeng, and F. R. Liang, "History of acupuncture research," in International Review of Neurobiology, vol. 111, chapter 1, pp. 1-23, Elsevier, 2013.

[2] J. G. Lin, C. H. Chen, Y. C. Huang, and Y. H. Chen, "How to design the control group in randomized controlled trials of acupuncture?" Evidence-Based Complementary and Alternative Medicine, vol. 2012, Article ID 875284, 7 pages, 2012.

[3] World Health Organization, "WHO Traditional Medicine Strategy: 2014-2023," [EB/OL], http://apps.who.int/iris/bitstream/ 10665/92455/1/9789241506090_eng.pdf?ua=1, 2017.

[4] F. Liang, X. Yang, X. Wu, and Y. Tang, "Current situation and outlook of acupuncture clinical trials in the past 20 years in China," Shanghai Journal of Acupuncture and Moxibustion, vol. 26, no. 12, pp. 36-38, 2007.

[5] W. He, X. Wen, Y. Tong, X. Zhao, and Y. Li, "Quantitative analysis of acupuncture clinical trials in the past 10 years in China," Shanghai Journal of Acupuncture and Moxibustion, vol. 33, no. 4, pp. 375-376, 2014.

[6] X. Bai and X. Tian, "Clinical current situation and outlook of acupuncture and moxibustion," Journal of Changchun University of Traditional Chinese Medicine, vol. 28, no. 4, pp. 732-735, 2012.

[7] "About the WHO ICTRP," [EB/OL], http://www.who.int/ ictrp/about/en/, 2017.

[8] T. Wu, Y. Li, J. Li, and G. Li, "A milestone for clinical trials: the global clinical trial registration system lauched up," Chinese Journal of Evidence-Based Medicine, vol. 7, no. 7, pp. 479-480, 2007.

[9] D. Ghersi and T. Pang, "From Mexico to Mali: Four years in the history of clinical trial registration," Journal of Evidence-Based Medicine, vol. 2, no. 1, pp. 1-7, 2009.

[10] T. Evans, M. Gülmezoglu, and T. Pang, "Registering clinical trials: An essential role for WHO," The Lancet, vol. 363, no. 9419, pp. 1413-1414, 2004.

[11] R. F. Viergever and D. Ghersi, "The quality of registration of clinical trials," PLoS ONE, vol. 6, no. 2, Article ID e14701, 2011.

[12] K. Krleža-Jerić, "Clinical trial registration: The differing views of industry, the WHO, and the Ottawa Group," PLoS Medicine, vol. 2, no. 11, article e378, pp. 1093-1097, 2005.

[13] "WHO international clinical trials registry platform," [EB/OL], http://apps.who.int/trialsearch/,2017.

[14] “WHO Trial Registration Data Set (TRDS)," [EB/OL], http:// www.who.int/ictrp/network/trds/en/, 2017.

[15] T. Wu, T. Li, Z. Bian et al., "Practice Good Publication Practice to Improve the Transparence of Clinical Trials," Chinese Journal of Evidence-Based Medicine, vol. 6, no. 3, pp. 153-156, 2006. 
[16] I. Lund, J. Näslund, and T. Lundeberg, "Minimal acupuncture is not a valid placebo control in randomised controlled trials of acupuncture: a physiologist's perspective," Chinese Medicine, vol. 4, article 1, 2009.

[17] H. MacPherson, D. G. Altman, R. Hammerschlag et al., "Revised STandards for Reporting Interventions in Clinical Trials of Acupuncture (STRICTA): extending the CONSORT statement," PLoS Medicine, vol. 7, no. 6, Article ID e1000261, 2010.

[18] C. Cheng, S. Fu, Q. Zhou et al., "Extending the CONSORT Statement to moxibustion," Journal of Integrative Medicine, vol. 11, no. 1, pp. 54-63, 2013.

[19] K. F. Schulz, D. G. Altman, and D. Moher, "CONSORT 2010 statement: updated guidelines for reporting parallel group randomised trials," BMC Medicine, vol. 8, article 18, 2010.

[20] Y. Chae, "The dilemma of placebo needles in acupuncture research," Acupuncture in Medicine, vol. 35, no. 5, pp. 382-383, 2017.

[21] L.-L. Zhang, Q. Chu, S. Wang, H. Lai, and B.-B. Xie, "Is sham acupuncture as effective as traditional Chinese acupuncture? It's too early to say," Chinese Journal of Integrative Medicine, vol. 22, no. 7, pp. 483-489, 2016.

[22] N. P. Assefi, K. J. Sherman, C. Jacobsen, J. Goldberg, W. R. Smith, and D. Buchwald, "A randomized clinical trial of acupuncture compared with sham acupuncture in fibromyalgia," Annals of Internal Medicine, vol. 143, no. 1, pp. 10-19, 2005.

[23] A. Schneider, P. Enck, K. Streitberger et al., "Acupuncture treatment in irritable bowel syndrome," Gut, vol. 55, no. 5, pp. 649-654, 2006.

[24] D. Irnich, N. Salih, M. Offenbächer, and J. Fleckenstein, "Is sham laser a valid control for acupuncture trials?” Evidence-Based Complementary and Alternative Medicine, vol. 2011, Article ID 485945, 8 pages, 2011.

[25] Y. Liu, "Research on the Quality of Systematic Reviews and Randomized Controlled Trials of Acupuncture and Cognition of Reporting Guideline," Lanzhou University, 2012.

[26] "Acupuncture-Moxibustion Clinical Trial Registry (AMCTR)," [EB/OL], http://www.acmctr.org/, 2017. 


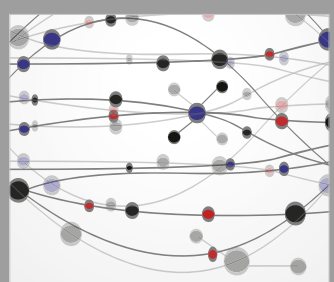

The Scientific World Journal
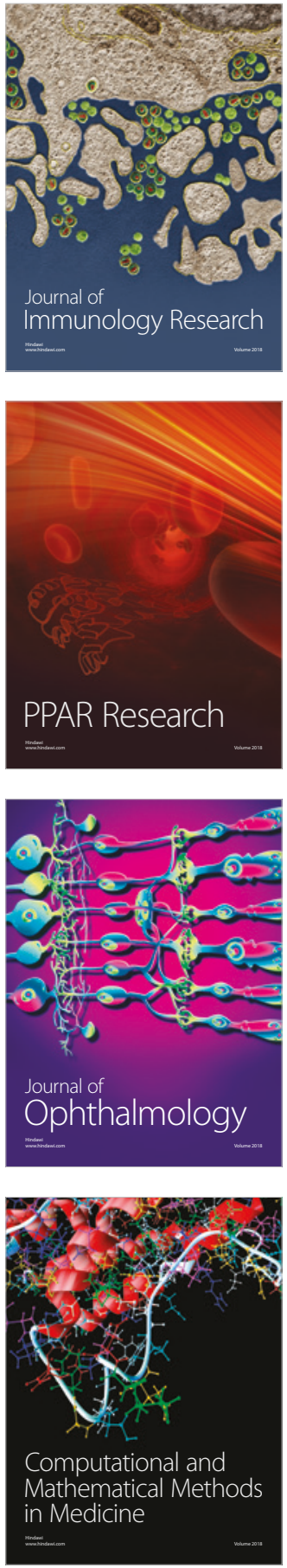

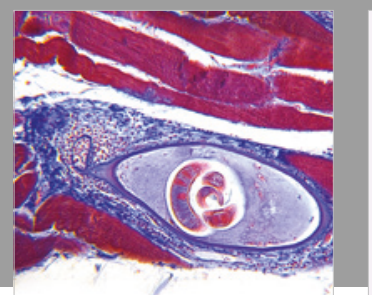

Gastroenterology Research and Practice

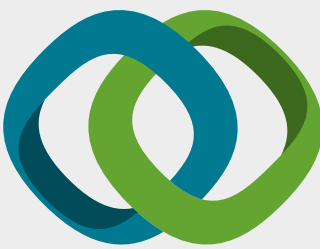

\section{Hindawi}

Submit your manuscripts at

www.hindawi.com
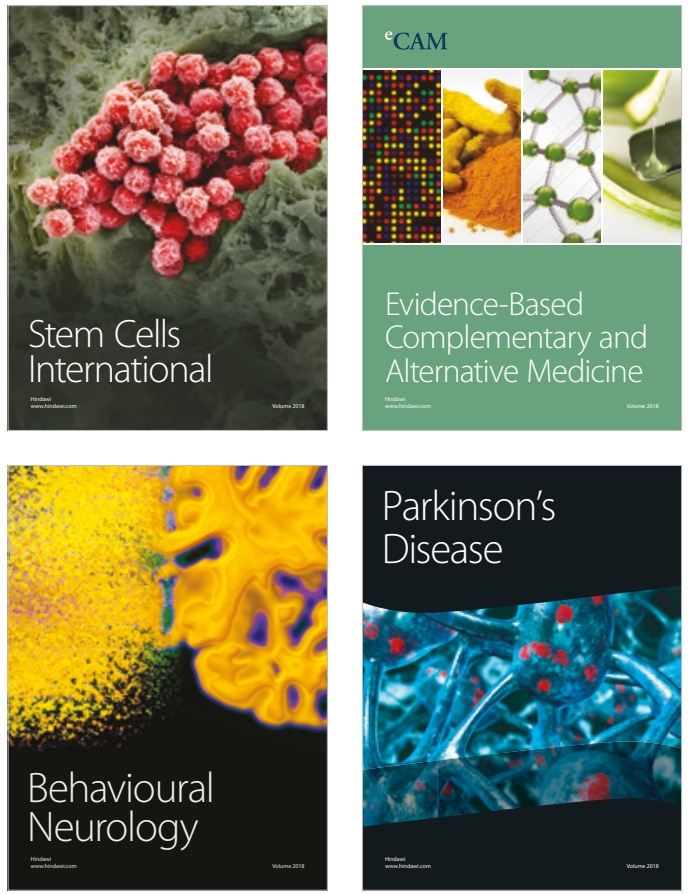

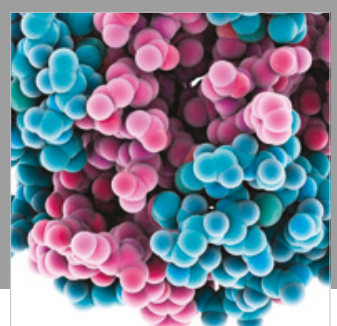

ournal of

Diabetes Research

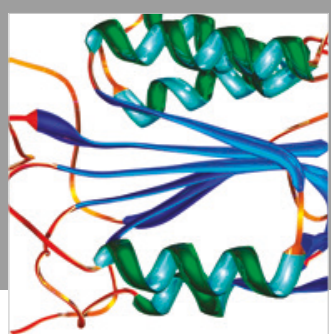

Disease Markers
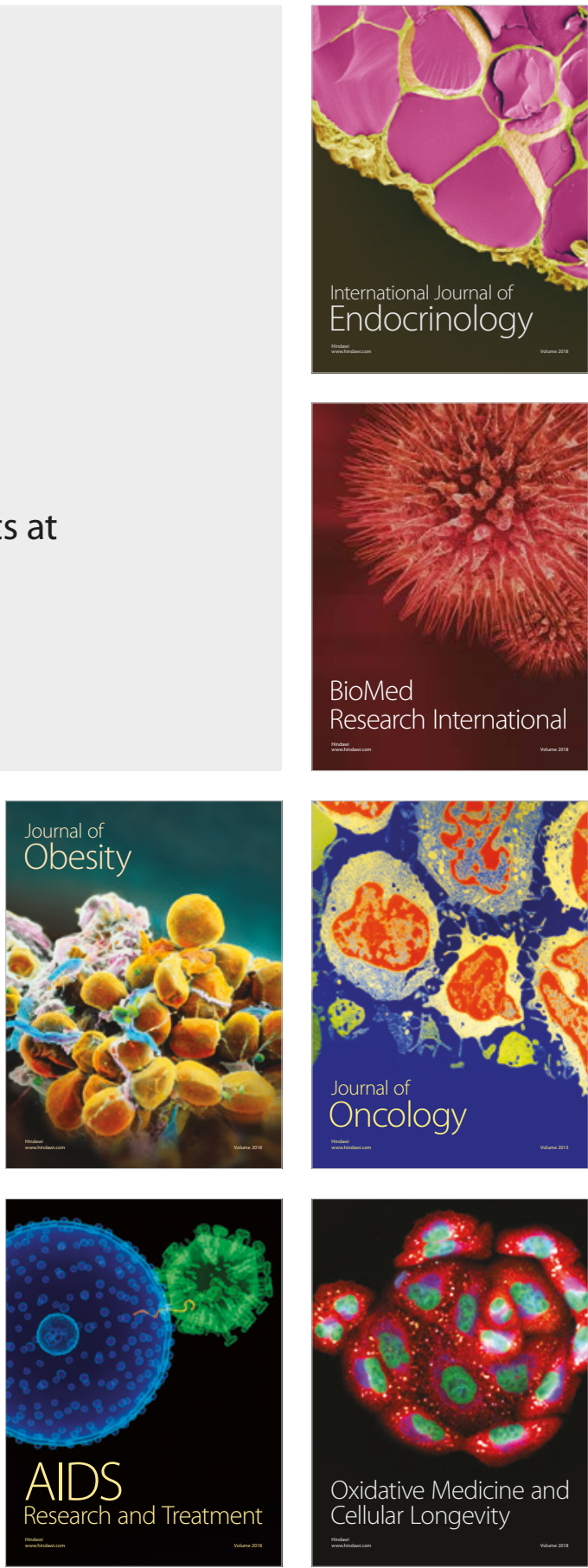